\title{
SHP solution of effect of elevated temperature on tunnel lining
}

\author{
P. Procházka \& S. Peskova \\ CTU Prague, Civil Engineering, Prague, Czech Republic
}

\begin{abstract}
Smooth hydrodynamic particle solution seems to be a suitable method for solving problems of influence of high temperatures on the FR concrete tunnel lining and surrounding rock. This meshless method suffers from one unpleasant property: it is not easy to describe the inhomogeneous geometrical boundary conditions. In our case, the highly elevated temperature is increasing and decreasing in a time scale on the boundary. After reformulating the problem into a form obeying boundary conditions being suitable for application of the SPH method, the 2D problem can be solved. This restriction to 2D is an impact of assumption that the fire is triggered along the length of the axial direction of tunnel. This follows from the observation at concrete locations where conflagrations inside of the tunnels took part. The most extreme temperature is prevailingly considered at $1200^{\circ} \mathrm{C}$ to fulfill European standards. As the mechanical and physical properties change nonlinearly a set of experiments in furnaces have been conducted to improve the characteristics of the material of tunnel lining and rock. Coupled modeling is applied to conduct convergence analysis providing results from experiments and numeric method to be in compliance. Simultaneous nonlinear equations are first formulated, involving stress analysis, influence of pore pressure, change of temperature, moisture, and degree of saturation. Nonlinear mechanical properties are based on the change of mechanical parameters, as are available from experimental studies. A couple of examples will follow the theory.
\end{abstract}

Keywords: smooth particle hydrodynamics method, meshless approach, elevated temperature, tunnel lining and surrounding rock, partition of unity. 


\section{Introduction}

The problem of fire defense of tunnel linings stems from the early beginning of tunnel construction. In an age where vehicles are now powered by explosive fuel, used both in passenger vehicles and freight transport, if an accident occurs then there can be a disastrous aftermath to the tunnel lining and sometimes also to the surrounding rock both of which can lead to the supporting system of such tunnels to become no longer serviceable. A couple of experimental studies are published on this topic, although until today experiments of this kind appear to be relatively very expensive. The experiments show the overall properties, failure strength of material, surface cracking, and other phenomena, which can be seen from the outside of the sample tested. Of greater interest to researchers and engineers is the knowledge about the process developing inside the sample, mainly the reaction of the material to sudden change of temperature on the boundary of the sample. This can be solved by some appropriate numerical methods. One of the most appropriate for this problem seems to be smoothed particle hydrodynamics (SPH), which belongs to mesh-free methods, historically developed for astrophysical applications, $[1,2]$. The inherent benefit of the SPH formulation consists in transformation of partial differential equations to a system of linear algebraic using regularization. This transformation is, among others, suitable for parallel computations. Recently, SPH has grown into a successful and respected numerical tool. In particular, this method does not differ between 3D, 2D and 1D problems, as the problems defined in higher order spaces can be simulated as easy as that in 1D. An excellent review of the advantages and recent progress in SPH can be found in [3, 4]. Some problems occur when geometrical boundary conditions should be involved. Takeda et al. [5] proposed ghost particle method, in which some particles are located outside the domain. Heat conduction problem are solved in [6], where Taylor series expansion approximates the regularization kernels.

\section{Regularization of functions and their derivatives}

The concept behind SHP is based on an interpolation scheme. From mathematical calculus it is well known, [7], that for each generalized function $f$ defined on a domain $V \subset R^{n}$ with boundary $S$ there exists a positive $\varepsilon$ and a finite cover $\{\Omega\}_{i=1}^{N} \subset V, i=1, \ldots, N$ (for each point $x \in V$ there is an index $i \in 1, \ldots, N$ so that $x \in \Omega_{i}$ ) with measure of $\Omega_{i}<\varepsilon$ so that on $\Omega_{i}$ there exists function $\omega_{\varepsilon}^{i} \in C^{\infty}(\bar{\Omega})$, supp $\omega_{\varepsilon}^{i} \in \Omega_{i}$ (sometimes called cap function) which regularize the function $f$ in such a way that $f$ can be expressed as

$$
f(\boldsymbol{x})=\sum_{i=1}^{N} \int_{\Omega_{i}} f(\boldsymbol{\xi}) \omega_{\varepsilon}^{i}(\boldsymbol{x}-\boldsymbol{\xi}) \mathrm{d} \boldsymbol{\xi}=f * \omega_{\varepsilon}^{i}, i=1, \ldots, N,
$$


and the left hand side of the latter relation is called the regularization, $f^{*} \omega_{\varepsilon}^{i}$ is the convolution. Recall some basic properties of the regularization: the volume of each cap function is unity, is equal to one. If the function $f$ is uniform (equal to one) and $\varepsilon \rightarrow$ infinity the regularization turns to be density of the function $f$, for example density of probability. If $\varepsilon \rightarrow 0$ the kernel $\omega_{\varepsilon}^{i}$ turns to be the Dirac function. For each positive $\varepsilon$ the regularization (kernel, cap function) $\omega_{\varepsilon}^{i}$ can be created infinitely differentiable (for definition of types of cap functions, see [7], for example).

Since different cap functions should be created for different $\Omega_{i}$, the above definition becomes inconvenient. In order to improve this put $\omega_{\varepsilon}^{i} \equiv \omega_{\varepsilon}$ and the shape of $\Omega_{i}$ remains same for all $i$, the area of a circle in $2 \mathrm{D}$ or the volume of a sphere, for example. Now inside of the domain $V$ select a set of points $\boldsymbol{x}_{i}, i=1, \ldots, N, \boldsymbol{x}_{i}$ is centered at $\Omega_{i}$ and a new function $F$ is defined as

$$
F\left(\boldsymbol{x}_{i}\right)=\sum_{i=1}^{N} \int_{\Omega_{i}} f(\boldsymbol{\xi}) \omega_{\varepsilon}\left(\boldsymbol{x}_{i}-\boldsymbol{\xi}\right) \mathrm{d} \boldsymbol{\xi}, i=1, \ldots, N
$$

which is formally similar to relation (1), so that it fulfils basic properties above mentioned. Since the former assumptions take place the function $F$ cannot be expected to be equal to $f$ any longer, but a special case: $\varepsilon \rightarrow 0$ in the sense of definition of the Dirac function.

In our case 2D problem is considered and degrees of freedom are concentrated at nodes $\boldsymbol{x}_{i} \in \Omega_{i}, i=1, \ldots, N, \Omega_{i}$ are considered as areas of the circles in which $x_{i}$ is centered. In the approximation, the smoothed (regularized) function $F$ for any physical quantity $f$ is identified with the original function, i.e. $F \equiv f$. Moreover, the kernel $\omega_{\varepsilon}$ is simplifies for real calculations and the simplification is denoted as $W_{\varepsilon}$. Introducing this to (2) and setting $f_{i}=f\left(\boldsymbol{x}_{i}\right)$ gives:

$$
f_{i}=f\left(x_{i}\right)=\int_{\Omega_{i}} f(\boldsymbol{\xi}) W_{\varepsilon}\left(\boldsymbol{x}_{i}-\boldsymbol{\xi}\right) \mathrm{d} \boldsymbol{\xi}
$$

Equation (3) is the kernel representation to average functional distribution. In our next considerations additional properties of $W_{\varepsilon}$ will be required:

$$
\begin{array}{cc}
\text { positivity: } & W_{\varepsilon}\left(\boldsymbol{x}_{i}-\boldsymbol{\xi}\right) \geq 0, \quad \boldsymbol{\xi} \in \Omega_{i} \\
\text { normalization: } & \int_{\Omega_{i}} W_{\varepsilon}\left(\boldsymbol{x}_{i}-\boldsymbol{\xi}\right) \mathrm{d} \boldsymbol{\xi}=1, \quad \forall \varepsilon>0 \\
\text { surface smoothness on } \partial \Omega_{i}: & \begin{array}{r}
W_{\varepsilon}\left(\boldsymbol{x}_{i}-\boldsymbol{\xi}\right)=\nabla W_{\varepsilon}\left(\boldsymbol{x}_{i}-\boldsymbol{\xi}\right)= \\
=\nabla \nabla W_{\varepsilon}\left(\boldsymbol{x}_{i}-\boldsymbol{\xi}\right)=0, \quad \boldsymbol{\xi} \in \partial \Omega_{i}
\end{array}
\end{array}
$$

The last property follows from the fact that the order of differential equations, which are to be studied, is two, and so is the required regularity (continuity). 
Using integration by parts, from the boundary conditions on $\partial \Omega_{i}$ it immediately follows that

$$
\int_{\Omega} \nabla W_{\varepsilon}\left(\boldsymbol{x}_{i}-\boldsymbol{\xi}\right) \mathrm{d} \boldsymbol{\xi}=\int_{\Omega} \nabla \nabla W_{\varepsilon}\left(\boldsymbol{x}_{i}-\boldsymbol{\xi}\right) \mathrm{d} \boldsymbol{\xi}=0
$$

For the sake of simplicity the approximation of the kernel $W_{\varepsilon}$ is represented by

$$
W_{\varepsilon}\left(\boldsymbol{x}_{i}-\boldsymbol{\xi}\right)=C\left(1-10 r^{3}+15 r^{4}-6 r^{5}\right), \quad r=\left|\boldsymbol{\xi}-\boldsymbol{x}_{i}\right| / \varepsilon \quad \text { for } 2 \mathrm{D}
$$

where $C=\frac{2}{h}$ for $1 \mathrm{D}$ problem, $C=\frac{7}{\pi h^{2}}$ for $2 \mathrm{D}$ problem, and $\left|\boldsymbol{\xi}-\boldsymbol{x}_{i}\right|$ is the distance between the pertinent points.

If we consider volume (area, interval) of an element $\Omega_{i}=\frac{m_{i}}{\rho_{i}}$, where $m_{i}$ is the mass of the element and $\rho_{i}$ is the density, using rectangular rule of evaluation of integrals yields:

$$
f_{i}=f\left(x_{i}\right)=\sum_{r_{i j} \leq h} \frac{m_{j} f_{j}}{\rho_{j}} W_{\varepsilon}\left(r_{i j}\right), r_{i j}=\left|\boldsymbol{x}_{j}-\boldsymbol{x}_{i}\right|, \nabla f_{i}=\rho_{i} \sum_{r_{i j} \leq h} m_{j}\left(\frac{f_{i}}{\rho_{i}^{2}}+\frac{f_{j}}{\rho_{j}^{2}}\right) \nabla W_{\varepsilon}\left(r_{i j}\right)(6)
$$

\section{Governing equations}

According to [8], the governing equations for coupled heat-seepage-stress problem are listed as:

$$
\begin{gathered}
\frac{\partial \rho}{\partial t}=-\rho \frac{\partial v^{\alpha}}{\partial x^{\alpha}} \\
C \frac{\partial T}{\partial t}=\frac{\partial}{\partial x}\left(\lambda_{x} \frac{\partial T}{\partial x}\right)+\frac{\partial}{\partial y}\left(\lambda_{y} \frac{\partial T}{\partial y}\right)+\frac{\partial}{\partial z}\left(\lambda_{z} \frac{\partial T}{\partial z}\right)+ \\
+C_{w} \rho_{w}\left(V_{x} \frac{\partial T}{\partial x}+V_{y} \frac{\partial T}{\partial y}+V_{z} \frac{\partial T}{\partial z}\right) \\
S \frac{\partial p}{\partial t}=\frac{\partial}{\partial x}\left(K_{x} \frac{\partial p}{\partial x}\right)+\frac{\partial}{\partial y}\left(K_{y} \frac{\partial p}{\partial y}\right)+\frac{\partial}{\partial z}\left(K_{z} \frac{\partial p}{\partial z}\right)+Q \\
V_{x}=K_{x} \frac{\partial p}{\partial x}, \quad V_{y}=K_{y} \frac{\partial p}{\partial y}, \quad V_{z}=K_{z} \frac{\partial p}{\partial z} \\
K_{x}=K_{0 x} \exp \left(-\eta \sigma_{x}^{\mathrm{eff}}\right), K_{y}=K_{0 y} \exp \left(-\eta \sigma_{y}^{\mathrm{eff}}\right), K_{z}=K_{0 z} \exp \left(-\eta \sigma_{z}^{\mathrm{eff}}\right)
\end{gathered}
$$

where

$T, p, C$ and $\lambda$ are the temperature, the pressure, the volumetric heat capacity and the thermal conductivity of the lining and surrounding rock, respectively; 
$S, K$ are the water-supply degree and the hydraulic conductivity, respectively;

$\eta$ is a constant, received from appropriate tests;

$\sigma_{x}^{\text {eff }}, \sigma_{y}^{\text {eff }}$ and $\sigma_{z}^{\text {eff }}$ are the effective stresses in $x, y$ and $z$ directions, respectively;

$C_{w}$ and $\rho_{w}$ are the specific heat and the density, respectively, of water.

Equation (7) describes continuity, (8) is a diffusion equation for temperature distribution, (9) is a diffusion equation for pore pressure, and momentum equation has to be added to complete the system of the coupled problem:

$$
\frac{\partial v^{\alpha}}{\partial t}=-\frac{1}{\rho} \frac{\partial \sigma^{\alpha \beta}}{\partial x^{\beta}}
$$

the moving equation for particles is

$$
\frac{\partial u^{\alpha}}{\partial t}=v^{\alpha}
$$

where $u^{\alpha}=x_{n e w}^{\alpha}-x_{\text {old }}^{\alpha}$ i.e. a component of the displacement is difference between new and old values of the pertinent components of coordinates.

In the sense of previous explanation the above introduced strongly nonlinear equations can be solved using iteration at each time step. The way of development of temperature due to fire extent at the surface of tunnel lining is described from experiments. The only problem in applications of SPH appears to be inhomogeneous boundary conditions. At each time step we change the elliptic equation of the type

$$
A x=b \text { in } \Omega, x=\bar{x} \text { on } \Gamma,
$$

where $\Omega, \Gamma$ are domain and its boundary, into

$$
A \xi=c, \xi=x-\bar{x}, c=b-A \bar{x} \text { in } \Omega, \xi=0 \text { on } \Gamma .
$$

In the case of homogeneous boundary conditions being prescribed, the problem is easily solvable. Note that the operator $A$ can be considered linear at each time step (it actually is pseudo-linear).

\section{Young's modulus}

The spalling of the concrete lining during combustion in a tunnel and deterioration of the material properties is not easy to obtain from experiments. The only information, which appears to be reliable, is the distribution of Young's modulus in relation to the temperature. Also, this information is not quite precise as it depends on the position of a point in which the measurement takes part inside the lining. There are a couple of authors who tried to express the relation between the values of Young's modulus and higher temperature. Note that the temperature is considered between room temperature and $1200^{\circ} \mathrm{C}$, according to European standards [9]. 
The test results of elastic modulus of concrete at high temperature are compared in [10]. It can be noted that, except for HPC, the elastic modulus decreases monotonically as the temperature increases, unlike the behavior of the compressive strength. Generally speaking, the elastic modulus has no or a little effect till temperature of $70^{\circ} \mathrm{C}$ after that it descends approximately in a linear way with the increase of the temperature. The secant modulus at the stress of $0.4 f_{c}^{T}$ was assumed as the elastic modulus by $\mathrm{Lu}$ [11] and tri-linear expression between $E_{c}{ }^{T}$ and the temperature $T$ was given as follows:

$$
E_{c}^{T}=\left\{\begin{array}{lr}
(0.1-0.0015 T) E_{c} & 20^{\circ} C \leq T \leq 300^{\circ} C \\
(0.87-0.00087 T) E_{c} & 300^{\circ} C \leq T \leq 700^{\circ} C \\
0.28 E_{c} & T>700^{\circ} C
\end{array}\right\}
$$

where $E_{c}{ }^{T}$ and $E_{c}$ are the elastic modulus of concrete at elevated and room temperature, respectively.

Xiao and König [10], indicated that the elastic modulus of HPC degraded much more obviously than that of NSC below $400^{\circ} \mathrm{C}$, while above $400^{\circ} \mathrm{C}$ its degradation was almost the same as that of NSC. It was also reported in $[12,13]$ that the type of aggregate would influence the modulus of elasticity distinctively and $E_{c}{ }^{T}$ could decrease with the increase of water-cement ratio as well. The elastic modulus at elevated temperature drops much more greatly for concrete cured in water than that of cured in the air. The deterioration of elastic modulus seems to have nothing to do with the heating-cooling cycles but is related to the maximum elevated temperature during heating $[14,15]$.

\section{Example}

The problem of the heating of a lining on the upper side of the tunnel in symmetric angle of $90^{\circ}\left(45^{\circ}\right.$ on each size of the vertical axis) by a temperature of $1200^{\circ} \mathrm{C}$ is solved. Standard fiber reinforced concrete with basalt fibers is considered, while the surrounding rock possesses material properties of class G4, which provides:

$$
E=1000 \mathrm{MPa}, E_{\text {plast }}=800 \mathrm{MPa}, E_{\text {residual }}=500 \mathrm{MPa}, v=0.25, v_{\text {plast }}=0.46,
$$

$\varphi=25^{0}, C=100 \mathrm{MPa}$, where the former quantities describe elastic modulus, modulus at plastic state, its residual value, elastic Poisson's ratio, the same at plastic state, coefficient of internal friction, and shear strength (cohesion). For completeness the material of lining is considered as:

$$
E=27000 \mathrm{MPa}, E_{\text {plast }}=10000 \mathrm{MPa}, E_{\text {residual }}=5000 \mathrm{MPa}, v=0.2,
$$
$v_{\text {plast }}=0.46, \varphi=35^{\circ}, C=150 \mathrm{MPa}$. The density of the concrete is $27 \mathrm{kN} / \mathrm{m}^{3}$, the density of the rock is $33 \mathrm{kN} / \mathrm{m}^{3}$.

Since both geometric and loading symmetry is obvious, the structure is solved as symmetric. In Fig. 1 hypsography of horizontal stresses in a cut of a half of the domain are presented. The peak stresses are concentrated to the upper and lower part of the lining, while at the center of the lining they almost disappear. As the values of stresses are much larger in the lining then in the rock, in the cut 
from the domain of rock there is no change of horizontal stresses registered. In the figure the points at which the variables are calculated are marked. The maximum stress is observed on the vertical axis of symmetry and possesses the value of $10 \mathrm{MPa}$ in the lining.

Figure 2 shows the hypsography of normal horizontal stresses for the distribution of temperature $1200^{\circ}$ at the upper face of the lining (the same loading scheme as in the starting situation) after thirty minutes. Note that ten minute intervals are contemplated in which tests of plastic and damage states in

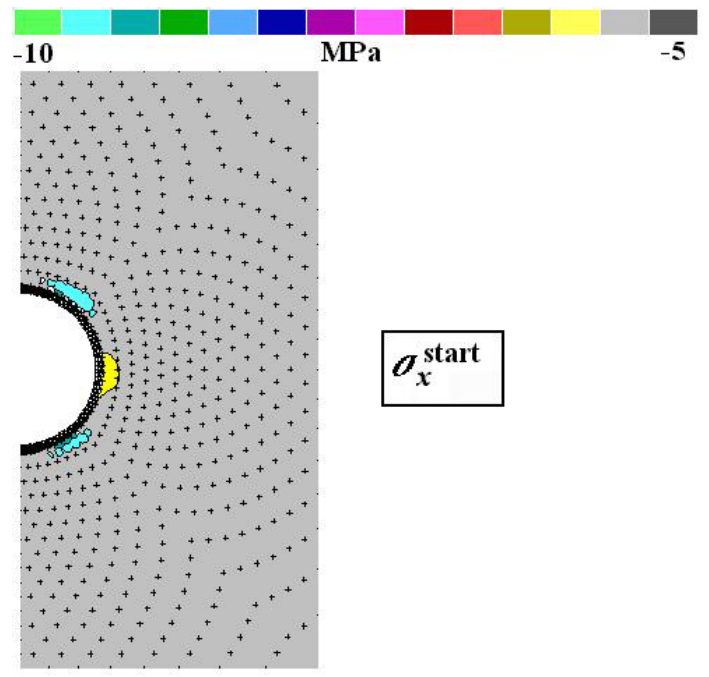

Figure 1: Horizontal stresses before fire.

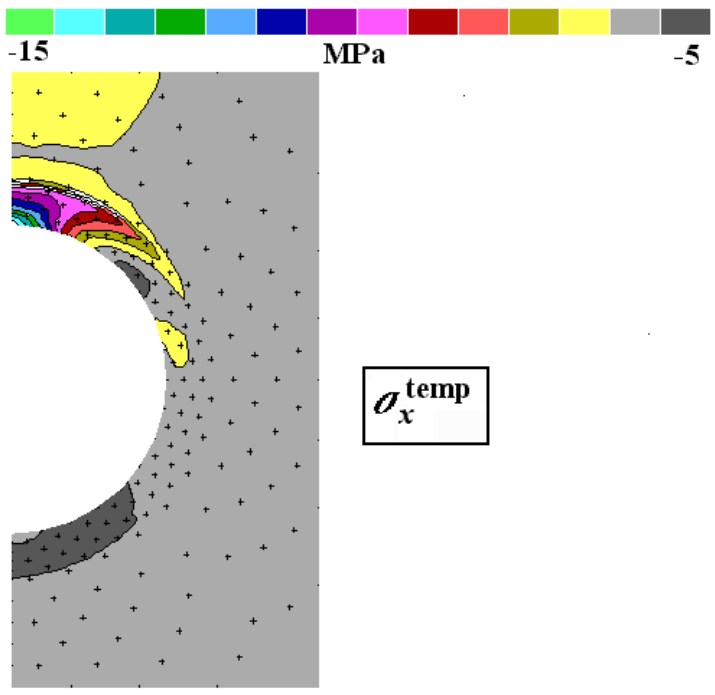

Figure 2: Horizontal stresses after half an hour combustion. 
the material of both lining and rock are carried out. The latter picture is enlarged in order to see in more details the behavior of stresses $\sigma_{x}$ in the neighborhood of the fire.

It is obvious from the figure that extreme stresses of $15 \mathrm{MPa}$ are attained at the calotte (vault) of the tunnel, where the combustion is concentrated. It is worth noting that after approximately two hours of fire the concrete looses its bearing capacity and the rock takes over the loading. Rock can also be affected by the fire and damage locally. In such conditions the repair and recovery of the tunnel can be a complex task takes a long time.

\section{Conclusions}

In this paper the combustion of the concrete lining and the rock surrounding the tunnel is solved by the Smooth Particle Hydrodynamics Method. The results are partly compared with experiments on concrete slabs burned in a furnace. Particularly, influences of fibers from fused basalt are observed and the composite with the concrete matrix is evaluated after carrying out tests in Innsbruck University. The basalt material seems to be quite suitable for this case of endangered concrete linings. The reason is that at lower temperatures it behaves as a stiffener of the concrete matrix and overcoming the temperature of $1000^{\circ} \mathrm{C}$ it becomes molten and enables vapor to escape from the concrete and not cause any larger damage. Consequently, in comparison to other types of fibers, fused basalt serves in a proper way. Natural fibers are very suitable for high temperatures (they burn out and the vapor can freely dilute in the air) and stiff fibers like steel serve as a good reinforcement but are too troublesome in the case of influence of high temperatures.

The SPH method is perfectly suitable for solving such a problem. This method shows very promising time consumption of computer (it is basically low in comparing it with other numerical methods), but generally it suffers from one undesirable property: inhomogeneous geometrical boundary conditions can be respected in the calculus in a complicated way. In our case this type of boundary conditions does not play any decisive role. The boundary conditions are declared by time changing the source of heat, and the damage in the material is calculated at the time-stage.

\section{Acknowledgements}

Work on this project has been financially supported by GACR, project No. $103 / 06 / 1124$. Sponsorship of CIDEAS is also acknowledged.

\section{References}

[1] Lucy, L.B. (1977). A numerical approach to testing of the fission hypothesis. Astron. J. 82, 1013.

[2] Gingold, R.A. and Monoghan, J.J. (1977). Smooth particle hydrodynamics: theory and application to non-spherical stars. Monthly Nat. R. Astron. Soc. 181, 375 . 
[3] Randle, P.W. and Libersky, L.D. (1996). Smooth particle hydrodynamics: some recent improvements and application. Appl. Mech. Engng. 139, 175.

[4] Li, S. and Liu, W.K. (2002). Meshfree and particle method and their applications. Appl. Mech. Rev. 55, 1.

[5] Takeda, H., Miyama, S. and Sekiya, M. (1994). Numerical simulation of viscous flow by smoothed particle hydrodynamics. Prog. Theor. Phys. 92, 939.

[6] Chen, J.K., Beraun, J.E. and Carney, T.C. (1999). A corrective smooth particle method for boundary value problems in heat conduction. Int. J. Numer. Methods Engrg. 46, 231.

[7] Vladimirov, V.C. (1971). Equations of mathematical physics. Nauka, Moscow.

[8] Lai, Y.M., Wu, Z.W., Zhu, Y.L. and Zhu, L.N. (1998). Nonlinear analysis for the coupled problem of temperature, seepage and stress fields in coldregion tunnels. Tunneling and Underground Space Technology 13(4), 435.

[9] COMITE EURO-INTERNATIONAL DU BETON (CEB), "Fire design of concrete structure," CEB, Lausanne, July (1991).

[10] Xiao J. and König G. "Study on concrete at high temperature in China-an overview". Fire Safety Journal, 39, 89-103(2004)10.

[11] Lu ZhD., "A Research on fire response of reinforced concrete beams" $\mathrm{PhD}$ thesis, Tongji University, (1989)

[12] Ding W.," Research and evaluation on repair of RC frame after fire by expert system", Master thesis, Tongji University, (1991).

[13] Wu B., Yuan J. and Wang GY., "Experimental study on the mechanical properties of HSC after high temperature" Chin J Civil Eng.,33, 8-15 (2000).

[14] Hu B. L., Song Y. P. and Zhao GF., "Test on strength and deformation of concrete under complex stress at elevated temperature", Build Sci Res Sichuan, 20, 47-50 (1994).

[15] Li W. and Guo ZhH., "Experimental investigation on strength and deformation of concrete under high temperature". Chin. J Build Struct. 14, $8-16$. 